\title{
1 Development of migrating entheses involves replacement of progenitor populations
}

2 Neta Felsenthal ${ }^{1}$, Sarah Rubin ${ }^{1}$, Tomer Stern ${ }^{1}$, Sharon Krief ${ }^{1}$, Deepanwita Pal $^{2}$, Brian A. Pryce ${ }^{2}$, Ronen

3 Schweitzer $^{2}$ and Elazar Zelzer ${ }^{1}$

4

$5{ }^{1}$ Department of Molecular Genetics, Weizmann Institute of Science, Rehovot 76100, Israel

$6 \quad{ }^{2}$ Research Division, Shriners Hospital for Children, Portland, OR 97201, USA

7

8 Corresponding author: eli.zelzer@weizmann.ac.il

9

10

11 Keywords: Musculoskeletal development, Enthesis, Sox9, Gli1, Progenitor cell, Hedgehog signaling,

12 R26R-Confetti 
14 ABSTRACT

15 Attachment sites of tendons to bones, called entheses, are essential for proper musculoskeletal function.

16 They are formed embryonically by $\operatorname{Sox} 9+$ progenitors and undergo a developmental process that

17 continues into the postnatal period and involves Glil lineage cells. During bone elongation, some

18 entheses maintain their relative positions by actively migrating along the bone shaft, while others,

19 located at the bone's extremities, remain stationary. Despite their importance, we lack information on

20 the developmental transition from embryonic to mature enthesis and on the relation between Sox9+

21 progenitors and Glil lineage cells. Here, by performing a series of lineage tracing experiments, we

22 identify the onset of Glil lineage contribution to different entheses during embryogenesis. We show that

23 Glil expression is regulated by SHH signaling during embryonic development, whereas postnatally it is

24 maintained by $\mathrm{IHH}$ signaling. Interestingly, we found that unlike in stationary entheses, where $\operatorname{Sox} 9+$

25 cells differentiate into the Gli1 lineage, in migrating entheses the Sox9 lineage is replaced by Gli1

26 lineage and do not contribute to the mature enthesis. Moreover, we show that these Glil+ progenitors

27 are pre-specified embryonically to form the different cellular domains of the mature enthesis.

28 Overall, these findings demonstrate a developmental strategy whereby one progenitor population

29 establishes a simple, embryonic tissue, whereas another population is responsible for its maturation into

30 a complex structure during its migration. Moreover, they suggest that different cell populations may be

31 considered for cell-based therapy of enthesis injuries. 


\section{INTRODUCTION}

The proper assembly of the musculoskeletal system is essential for the function, form and stability of the organism. During embryogenesis, an attachment between tendon and bone, known as enthesis, is formed. Thereafter and into the postnatal period, the rudimentary enthesis further develops into a more complex tissue. While some knowledge on enthesis formation and maturation exists, far less is known about the processes that transform the simple embryonic enthesis into the structure of the mature enthesis.

Traditionally, entheses are divided to either fibrous or fibrocartilaginous, according to their composition. Fibrous entheses form at the attachment site of a tendon that is inserted directly into the bone shaft, forming a structure that resembles a root system. This structure, which is composed of a dense connective tissue (Doschak and Zernicke, 2005; Shaw and Benjamin, 2007; Wang et al., 2013), was shown to be regulated by parathyroid hormone-like hormone (PTHLH, also known as PTHrP) (Wang et al., 2013). Fibrocartilage entheses typically form at attachment sites of tendons to the epiphysis or to bone eminences. Relative to fibrous entheses, fibrocartilage entheses are structurally more complex, displaying a cellular gradient that is typically divided into four zones, namely tendon, fibrocartilage, mineralized fibrocartilage and bone. The graded tissue that develops postnatally dissipates the stress that forms at the attachment site, thereby providing the enthesis with the mechanical strength necessary to withstand compression (Benjamin et al., 2006).

Enthesis development is initiated by the specification of a specialized pool of progenitor cells that express both SRY-box 9 (Sox9), a key regulator of chondrogenesis, and the tendon marker scleraxis (Scx) (Akiyama et al., 2005; Blitz et al., 2013; Schweitzer et al., 2001; Sugimoto et al., 2013). Cell lineage studies showed that Sox9-expressing progenitor cells contribute to the formation of the enthesis in neonatal mice (Akiyama et al., 2005; Soeda et al., 2010). Another marker for enthesis cells is GLI- 
57 Kruppel family member GLI1 (Glil), a component of the hedgehog (HH) signaling pathway. Lineage

58 studies revealed that Glil-expressing cells act as progenitors that contribute to the formation of some adult entheses (Dyment et al., 2015; Schwartz et al., 2015). However, the relation between progenitors of the embryonic enthesis and Glil lineage cells, which contribute to the mature enthesis, has not been

61 determined.

62 The relative position of an enthesis along the bone directly affects its mechanical function and, 63 subsequently, the animal's mobility (Polly, 2007; Salton and Sargis, 2009). Recently, it was shown that 64 all entheses maintain their relative position during bone elongation (Stern et al., 2015). The mechanism that maintains their positions involves regulation of the relative growth rates at the two epiphyseal plates. Additionally, some entheses migrate through continuous reconstruction to maintain their position, a process known as bone modelling or drift (Benjamin and McGonagle, 2009; Dörfl, 1980a; Dörfl, 1980b). These entheses, referred to in the following as migrating entheses, face a unique developmental challenge. Unlike most organs and tissues, they must develop into a complex graded tissue while constantly drifting. This raises the question of whether the descendants of the embryonic enthesis

71 progenitors continue to serve as the building blocks during maturation of migrating entheses.

72 In this work, we identify the embryonic stage at which Glil lineage is initiated and demonstrate its contribution to the postnatal enthesis. We show that embryonic Glil expression is initially under the regulation of SHH. Later during postnatal development, Gli1 expression is maintained by IHH. Moreover, we show that Sox 9 lineage does not contribute to postnatal migrating entheses. Instead, Gli1 lineage cells replace the Sox 9 lineage cells and populate the enthesis. Finally, we show that embryonic

77 Glil lineage cells are pre-determined to contribute to the different layers of the fibrocartilaginous 78 enthesis. 


\section{RESULTS}

81

82

\section{Some entheses undergo cellular and morphological changes while migrating}

The development of the rudimentary embryonic attachment site into the complex structure of a mature enthesis has received little attention (Galatz et al., 2007). As mentioned, in addition to substantial cellular and morphological changes, some entheses also migrate considerably along the bone during bone growth (Benjamin and McGonagle, 2009; Dörfl, 1980a; Dörfl, 1980b; Stern et al., 2015). Therefore, to study the transition that migrating entheses undergo during maturation, we documented morphological and molecular changes as well as drifting activity in entheses from embryonic day (E) 14.5 to postnatal day (P) 14. We focused on two migrating entheses, namely the deltoid enthesis (DT), a fibrocartilaginous enthesis that forms between the deltoid tendon and the deltoid tuberosity, and the teres major enthesis (TM), a fibrous enthesis that forms between the teres major tendon and the humeral shaft. Analysis of enthesis positions during development revealed that both DT $(0.778 \pm 0.026 \mathrm{~mm})$ and TM $(1.624 \pm 0.171 \mathrm{~mm})$ entheses drifted considerably along the bone shaft during bone elongation (Fig. 1A). Histological sections through wild-type (WT) mouse humeri showed that both embryonic entheses displayed a simple structure of layered cells (Fig. 1B,C(a),D(a)). However, the overall shape of the enthesis dramatically changed through development, as it protruded outwards from the bone shaft and the different enthesis domains became more noticeable (Fig. 1C(a'-a','),D(a'-a','). Tissue complexity also increased, as a larger variety of cells, such as fibrocartilage cells and osteoblasts (Benjamin and McGonagle, 2009), were identified along with an increase in extracellular matrix (Fig. 1C(d,d',h,h'), $\left.\mathrm{D}\left(\mathrm{d}, \mathrm{d}^{\prime}, \mathrm{h}, \mathrm{h}^{\prime}\right)\right)$. The increased complexity was also demonstrated by a change in the expression patterns of structural genes, such as bone sialoprotein (Bsp; also known as integrin binding sialoprotein (Ibsp), collagen type 2 alpha 1 (Col2al), collagen type 12 alpha 1 (Col12al) and tenascin C (Tnc), and regulatory genes such as Glil. Furthermore, expression domains correlating to various structural 
domains emerged, namely Coll2al, Tnc, Glil and Collal in tendon and fibrocartilage (Fig. 1C(c,fh),D(c,f-h), and Collal, Glil and Bsp in mineralized fibrocartilage and bone (Fig. 1C(c,e,h),D(c,e,h)).

\section{Gli1+ cell lineage contributes to the postnatal enthesis}

Previously, lineage tracing experiments showed that Glil lineage cells contribute to postnatal enthesis development (Dyment et al., 2015; Schwartz et al., 2015). Yet, the onset of this lineage during embryogenesis and its dynamics in different entheses have been missing. In order to identify the onset of Gli1 lineage, we performed pulse-chase experiments on Glil-CreER ${ }^{T 2}$ (Ahn \& Joyner, 2004) mice crossed with R26R-tdTomato reporter mice (Madisen et al., 2010), which allowed us to mark Gli1 expressing cells at specific time points and follow their descendants. We analyzed three entheses representing different types, namely DT (migratory-fibrocartilaginous), TM (migratory-fibrous), and Achilles (stationary-fibrocartilaginous). Examination of neonatal (P0) TM and DT entheses following tamoxifen administration at E11.5 and E12.5 revealed only a few Gli1 lineage cells. However, administration at E13.5 and E15.5 resulted in extensive labeling in both entheses (Fig. 2A-A','B-B','). In the stationary Achilles enthesis, extensive labeling was observed at P0 only after tamoxifen administration at E15.5 (Fig. 3C"'). The postnatal contribution of Glil lineage to migratory entheses was further established by following E13.5 lineage induction to P14 (Fig. 2D,E).

Together, these results indicate that although Glil lineage is not induced at the onset of enthesis formation (Blitz et al., 2013; Sugimoto et al., 2013), it contributes differentially to different entheses during embryonic development.

\section{Gli1 expression in migrating entheses is initiated by SHH and maintained by IHH}


The Hedgehog $(\mathrm{HH})$ signaling pathway has previously been suggested to play a role in regulating the activity of Gli1-positive enthesis cells (Breidenbach et al., 2015; Dyment et al., 2015; Liu et al., 2013; Schwartz et al., 2015). Thus, our finding that Glil expression is initiated at early stages of enthesis development (Fig. 1C(h)D(h)) raised the question of which component of the HH pathway regulates Glil expression in enthesis cells and whether it also affects migratory entheses. It was suggested that Indian hedgehog ( $\mathrm{IHH})$, an effector of the $\mathrm{HH}$ signaling pathway, is a possible regulator of Glil lineage cells in adult stationary entheses. In order to examine the possible role of IHH in inducing Glil expression in the embryonic enthesis, we blocked Ihh expression in the limb by using Prxl-Cre-Ihh ${ }^{-1-}$ mice. Interestingly, at E14.5 Glil was expressed in control and mutant entheses, suggesting that Glil expression in embryonic enthesis progenitors is not regulated by Ihh (Fig. 3A,A'). We therefore examined the possible role of another regulator of $\mathrm{HH}$ signaling, namely sonic hedgehog (SHH), by analyzing Glil expression by embryonic enthesis cells in Shh KO embryos (Shh-GFPCre $\left.{ }^{-/-}\right)$. Results showed that Glil expression dramatically decreased in the mutant entheses compared to the WT (Fig. 3B,B'), suggesting that $\mathrm{SHH}$ is necessary for the induction of Glil in embryonic enthesis cells.

That result was intriguing, because we observed that Glil was constantly expressed by both embryonic and postnatal enthesis cells (Fig. 1A(h-h",'),B(h-h",')), while Shh is expressed in the limb only during embryogenesis (Harfe et al., 2004). This raised the question of how Glil expression in the enthesis is maintained after the loss of Shh expression. To address the possibility that Ihh controls Glil expression in the postnatal enthesis even though it is not involved in the induction of Glil expression in embryonic enthesis progenitors, we analyzed Prxl-Cre-Ihh ${ }^{-/}$mice at P10. Results showed that although Glil expression was maintained in mutant muscle and bone, in the enthesis it was completely lost (Fig. 3C,C'), indicating that $I h h$ is indeed required for the maintenance of Glil expression in enthesis cells. To identify the source of $I h h$ in the enthesis, we performed immunofluorescence staining for IHH 
148 protein in P6 enthesis sections. As seen in Figure 3 (D,D'), IHH was highly expressed in mineralized 149 fibrocartilage and bone regions at the enthesis center.

150 Taken together, these results suggest that Glil expression by embryonic enthesis progenitors is induced 151 by $\mathrm{SHH}$ and later, in the maturing enthesis, maintained by $\mathrm{IHH}$ originating in mineralized fibrocartilage 152 and bone.

\section{Sox9 lineage cells of the embryonic enthesis do not contribute to postnatal migrating entheses}

The embryonic enthesis originates from Scx/Sox9 double-positive progenitor cells (Blitz et al., 2013;

Sugimoto et al., 2013). Yet, the contribution of these progenitors to the postnatal enthesis and their relation to the Glil lineage cells have never been studied. To fill this gap, we first examined the contribution of Sox9 lineage to the postnatal enthesis. To that end, we performed a pulse-chase cell lineage experiment using mice that express Cre-ER under control of the Sox9 promoter (Sox9-CreER ${ }^{T 2}$ ) crossed with $R 26 R$-tdTomato reporter mice (Soeda et al., 2010). It was previously demonstrated that tamoxifen administration at E12.5 effectively labels embryonic enthesis cells (Blitz et al., 2013; Soeda et al., 2010). Indeed, examination at P0 following tamoxifen administration at E12.5 showed that the dramatic decrease in the contribution of tdTomato-expressing cells to the two migrating entheses (Fig. embryonic lineage, whereas in migrating entheses this lineage was lost. 
171 point, we repeated the experiment while administering tamoxifen at different time points from E11.5 to

172 E17.5 (Fig. 4B). Examination at P14 showed minimal contribution of Sox9 lineage cells to the postnatal

173 entheses, similar to the results obtained following pulsing at E12.5. Taken together, these results indicate 174 that the embryonic Sox9 lineage contributes poorly to postnatal migrating entheses, suggesting that these 175 entheses are populated by another cell lineage postnatally.

\section{Gli1 lineage cells replace the embryonic Sox9 lineage during enthesis maturation}

178 Our finding that both embryonic Glil and Sox9 lineages contribute to the stationary postnatal enthesis 179 suggests that these two genes mark a common cell lineage. Conversely, the finding that embryonic Sox9 180 lineage contributes to embryonic but not to postnatal migrating enthesis implies that postnatally, 181 embryonic Gli1 lineage replaces the Sox9 lineage to form the mature enthesis.

182 To study the process of lineage replacement and to follow its dynamics, we traced both lineages 183 throughout enthesis development, from E15.5 to maturation at P14, by pulse-chase experiments. As seen 184 in Figure 5, at E15.5 and P0 in both the DT and TM, Sox9 lineage cells populated the embryonic 185 enthesis. However, from P0 their number decreased dramatically and by P8, the enthesis contained only 186 a limited number of these cells. Concurrently, the number of cells of the Glil lineage gradually 187 increased in both entheses and, by P8, Glil+ cells inhabited most of the enthesis structure. Interestingly, 188 the gradual population of the DT and TM entheses by Glil lineage cells correlated with the temporal 189 dynamics of enthesis migration (Fig. 1A). These results support our hypothesis that during early 190 postnatal development of migrating entheses, a new population derived from Glil-positive progenitors 191 substitutes the Sox9 lineage cells of the embryonic enthesis.

\section{Cell fate of Glil-positive progenitors is predetermined}


194 The increasing cellular complexity of the developing enthesis and our finding that progenitors of the 195 Glil lineage that will contribute to the postnatal enthesis are present already during embryonic 196 development led us to ask how these cells form the graded tissue of the mature enthesis. Specifically, we 197 sought to determine whether Gli1 lineage cells possess a multipotent capacity and, thereby, produce the 198 different cell types of the various enthesis domains, or have predetermined cell fates already at the time 199 of their recruitment to the embryonic enthesis. To decide between these options, we conducted pulse200 chase experiments on Gli1-CreER $R^{T 2}$ mice crossed with R26R-Confetti mice (Snippert et al., 2010) that, 201 upon recombination, stochastically express GFP, RFP, CFP or YFP, allowing for the identification of 202 different cell clones. Clones derived from multipotent progenitors would be spread among the different 203 domains, whereas predetermined progenitors would produce clones that are confined to specific 204 domains.

205 We focused on the two main domains of the DT enthesis, namely mineralized and non-mineralized 206 fibrocartilage, and on the border between entheseal mesenchymal cells and bone in the TM enthesis. 207 Examination of P14 limbs following tamoxifen administration at E13.5 revealed multiple clones in both 208 entheses, most of which were restricted to a specific domain (Fig. 6). These results suggest that at E13.5, 209 the cell fates of Glil lineage progenitors have already been determined.

\section{DISCUSSION}

214 The transition from the embryonic to the mature enthesis has been understudied. Here, we use genetic 215 lineage tracing to unravel the cellular developmental sequence of fibrous and fibrocartilaginous 216 entheses. We show that although Glil+ progenitors contribute to entheses of both types, their 
217 contribution differ greatly between stationary and migratory entheses. In stationary entheses, Glil+ 218 progenitors are descendants of Sox9+ progenitors that have established the embryonic enthesis. 219 However, in migrating entheses, a separate, pre-specified population of Gli1+ progenitors is recruited to 220 the embryonic enthesis, co-populates it alongside Sox9+ progenitors and, eventually, replaces them 221 during postnatal development.

222 As mentioned, fibrous and fibrocartilaginous entheses exhibit marked differences in structure and 223 composition (Benjamin et al., 2002). Notwithstanding the importance of this distinction, the observed 224 differences in cellular origin between stationary and migrating entheses calls for a revision of the 225 traditional classification and suggests that it should be taken into account whether an enthesis is "migratory" or "stationary".

227 Organ development can be mediated by several cellular mechanisms. In a linear mechanism, an 228 embryonic set of progenitors forms a primordium and then continues to proliferate and differentiate to 229 form the mature organ. Another mechanism is cell recruitment, during which cells are supplied to the 230 forming organ after primordium establishment. A third mechanism involves template replacement, in 231 which an initial template is formed by one type of cells to be later replaced by another cell population, 232 which will form the mature organ. Interestingly, in the musculoskeletal system there are examples of all 233 these modes of development. Muscles and joints develop through cell recruitment (Buckingham et al., 234 2003; Shwartz et al., 2016), whereas most of the skeleton develops through template replacement, 235 namely by endochondral ossification (Kronenberg, 2003). Here, we show that stationary entheses 236 develop linearly by embryonic Sox $9+$ progenitors that form the postnatal enthesis and later upregulate 237 the expression of Gli1. However, we show that migrating entheses develop through template 238 replacement, as Gli1+ progenitors replace the Sox9+ embryonic enthesis cells and form the mature 239 enthesis. 
Previous studies have shown not only that Glil is a marker for the forming enthesis, but that the HH pathway plays an active role in regulating enthesis maturation and regeneration (Breidenbach et al., 2015; Dyment et al., 2015; Liu et al., 2013; Schwartz et al., 2015; Schwartz et al., 2017). Ihh expression was identified in proximity to Gli1+ progenitor population in stationary entheses (Dyment et al., 2015; Liu et al., 2013; Schwartz et al., 2015). Moreover, loss-of-function of Smo, a componenet of the HH pathway, in $S c x$-expressing cells resulted in reduced mineralization of the enthesis (Breidenbach et al., 2015; Dyment et al., 2015; Liu et al., 2013; Schwartz et al., 2015). Yet, the question of which ligand of the $\mathrm{HH}$ pathway regulates the specification of Glil+ cells in the embryo and, specifically, in migrating entheses has remained open. Our results clearly suggest that Glil expression is regulated by both $\mathrm{SHH}$ and $\mathrm{IHH}$ during enthesis development. In the embryo, we show that $\mathrm{SHH}$, but not $\mathrm{IHH}$, signaling is essential for Glil expression in the enthesis, implicating SHH in Glil+ progenitor specification. However, during postnatal development, Ihh expression is vital for maintaining Glil enthesis expression 252 (Fig. 7A).

253 During endochondral ossification, IHH regulates hypertrophic chondrocyte differentiation and, thereby, 254 bone elongation (Vortkamp et al., 1996). It is therefore possible that by controling both these processes, 255 IHH coordinates enthesis migration with the concurrent bone elongation to preserve enthesis position 256 along the shaft. However, the signals that govern enthesis positioning and migration along the bone, 257 including the possible effect of IHH on these processes, are yet to be elucidated.

258 Another key question regards the mechanism that facilitates the replacement between Sox9+ and Gli1+ 259 cell populations. It is possible that the former cells die, while the latter cells proliferate and populate the 260 entire enthesis. A more attractive hypothesis is that during enthesis drift, Sox9+ cells are removed 261 together with eroded bone tissue by osteoclasts or other phagocytic cells. However, the replacement 
mechanism remains an open question. Moreover, whether the replacement process is the mechanism that

263 allows migrating entheses to maintain their relative position along the bone is yet to be determined.

264 Finally, our finding that Glil-expressing cells are pre-determined already in the embryo suggests the 265 existence of an earlier, yet unknown player in the specification of these progenitors. The discovery of 266 this early marker gene may enable the identification of multipotent enthesis cells, which may hold 267 therapeutic potential.

268 To conclude, our findings shed light on developmental linearity in organogenesis. Using the enthesis as 269 a model system, we identify two different strategies of development (Fig. 7). The first, found in 270 stationary entheses, is linear development where embryonic progenitors and their descendants contribute 271 to the entire process of enthesis development, from embryogenesis to maturity. Conversely, in migrating 272 entheses, another developmental strategy was identified where one type of progenitor cells form an 273 embryonic template, only to be later replaced by another cell lineage that contributes to the mature 274 organ.

\section{MATERIALS AND METHODS}

277 Animals

278 All experiments involving mice were approved by the Institutional Animal Care and Use Committee 279 (IACUC) of the Weizmann Institute. Histology was performed on BL6 mice.

280 For lineage tracing experiments, Sox9-Cre (Akiyama et al., 2005), Sox9-CreER (Soeda et al., 2010) and 281 Gli1-CreER ${ }^{T 2}$ (Ahn and Joyner, 2004, Jackson laboratories) mice were crossed with R26R-tdTomato

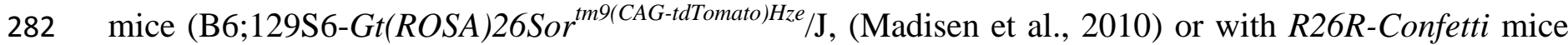




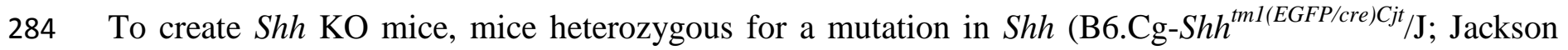

285 Laboratory) were intercrossed; heterozygotes or WT littermates were used as a control. The generation 286 of Prxl-Cre (Logan et al., 2002) was previously described. To generate Prxl-Cre-Ihh mutant mice, Ihh-

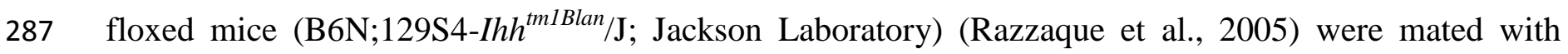
288 Prxl-Cre mice. As a control, Prxl-Cre-negative animals were used.

289 For cell lineage experiments, Sox9-CreER ${ }^{T 2 /+}$ or Glil-CreER ${ }^{T 2 /+}$ males were crossed with $R 26 R-$ 290 tdTomato females to produce embryos carrying both the relevant $C r e E R^{T 2}$ and $R 26 R$-tdTomato alleles. 291 For fate mapping, Glil-CreER ${ }^{T 2 /+}$ males were crossed with $R 26 R$-Confetti females to produce embryos 292 carrying both the Gli1-CreER ${ }^{T 2}$ and Confetti alleles.

293 In all timed pregnancies, plug date was defined as E0.5. For harvesting of embryos, timed-pregnant 294 females were sacrificed by cervical dislocation. The gravid uterus was dissected out and suspended in a 295 bath of cold phosphate-buffered saline (PBS) and the embryos were harvested after removal of amnion 296 and placenta. Tail genomic DNA was used for genotyping.

\section{Histological analysis, in situ hybridization and immunofluorescence}

299 For histology and in situ hybridization, embryos were harvested at various ages, dissected, and fixed in $4 \%$ paraformaldehyde (PFA)/PBS at $4^{\circ} \mathrm{C}$ overnight. After fixation, tissues were dehydrated to $70 \%$

$301 \mathrm{EtOH}$ and embedded in paraffin. The embedded tissues were cut to generate 7- $\mu \mathrm{m}$-thick sections and 302 mounted onto slides. Hematoxylin and eosin (H\&E) staining was performed following standard 303 protocols. Non-fluorescent and fluorescent in situ hybridizations were performed as previously 304 described using digoxigenin- (DIG) labeled probes (Shwartz and Zelzer, 2014). All probes are available 305 upon request. 
For immunofluorescence staining, 10- $\mu$ m-thick cryosections were air-dried for 1 hour before staining. For IHH staining, sections were washed twice in PBST for 5 minutes and blocked to prevent nonspecific binding with $7 \%$ goat serum and 1\% BSA dissolved in PBST. Then, sections were incubated with rabbit anti-IHH antibody (Abcam, \#AB39364, 1:50) at $4 \mathrm{C}^{\mathrm{O}}$ overnight. The next day, sections were washed twice with PBST and incubated for 1 hour with Cy2 conjugated fluorescent antibody (1:100, Jackson Laboratories). Slides were mounted with Immuno-mount aqueous-based mounting medium 312 (Thermo).

For Gli1 staining, 10- $\mu$ m-thick cryosections were air dried for 1 hour and fixed in 4\% PFA for 10 minutes. Then, sections were washed twice in PBST and endogenous peroxidase was quenched using $3 \% \mathrm{H}_{2} \mathrm{O}_{2}$ in PBS. Next, antigen retrieval was preformed using 0.3\% Triton in PBS. Non-specific binding was blocked using 7\% horse serum and 1\% BSA dissolved in PBST for 1 hour. Then, sections were

317 incubated with Goat anti-GLI1 antibody (R\&D systems, \#AF3455, 1:100) overnight at room 318 temperature. The next day, sections were washed twice in PBST and incubated with Biotin anti-goat (1:100, Jackson laboratories, 705065147) for 1 hour and then with streptavidin-HRP (1:200 Perkin Elmer, NEL750001EA) for 1 hour. HRP was developed using TSA amplification kit (1:100, Prekin 321 Elmer) for 20 minutes, counterstained with DAPI and mounted with Immuno-mount aqueous- based 322 mounting medium (Thermo).

\section{Physical position of entheses}

325 For identification of enthesis physical position, 2-4 limb samples at stages E16.5-P14 were scanned ex 326 vivo using iodine contrast agent to allow visualization of the soft tissue. DT and TM positions were 327 identified manually, and their physical position along the bone was calculated as described previously 328 (Stern et al., 2015). 


\section{Cell lineage analysis}

331 Tamoxifen (Sigma T-5648) was dissolved in corn oil (Sigma C-8267) at a final concentration of 5 $\mathrm{mg} / \mathrm{ml}$. Time-mated R26R-tdTomato females were administered $1 \mathrm{mg}$ of tamoxifen by oral gavage 333 (FST) at designated time points as indicated.

\section{Cell count}

336 For each age, 2-4 limbs from different litters were harvested, embedded in OCT, and sectioned at a 337 thickness of $10 \mu \mathrm{m}$. Sections were imaged using Zeiss LSM 780 microscope approximately every 80 $338 \mu \mathrm{m}$, capturing red and DAPI channels. Images were then processed in ImageJ as follows: Each image 339 was converted to an RGB stack and the region of interest was manually identified and cropped. Then, 340 image levels were adjusted to improve separation between nuclei. A binary threshold was set 341 automatically and conjoined nuclei were automatically separated using the binary watershed function. 342 Using a home-made Matlab script, the number of nuclei was counted. Each nucleus that was co343 localized with a red channel signal was counted as a tdTomato-positive cell. For each section, the 344 percentage of tdTomato-positive cells was calculated. The average percentage of these cells was 345 calculated first for each bone and then for all samples. From each line and for each age group, at least 3 346 individual bones were sampled, and 9-17 sections per bone were analyzed, depending on bone length. 347 Data are presented as mean $\pm \mathrm{SD}$.

\section{Fate mapping Confetti experiment}

350 Tamoxifen (Sigma T-5648) was dissolved in corn oil (Sigma C-8267) at a final concentration of 20 $351 \mathrm{mg} / \mathrm{ml}$. Time-mated R26R-Confetti females were administered $4 \mathrm{mg}$ of tamoxifen by oral gavage at 
352 E13.5. Cre-positive pups were sacrificed at P14. 1 hour prior to sacrifice, each pup was injected with

353 Calcein blue (Sigma, \#m1255; $30 \mathrm{mg} / \mathrm{kg}$ ). Limbs were harvested, fixed for 30 minutes in $4 \%$ PFA at $3544^{\circ} \mathrm{C}$, embedded in OCT, and sectioned at a thickness of $10 \mu \mathrm{m}$. Sections were imaged using Zeiss LSM 355780 microscope approximately every $80 \mu \mathrm{m}$.

\section{Microscope settings and image analysis}

358 At least 1024X1024 pixels, 8-bit images were acquired using the X20 lens. A Z-stack of 2-4 images was 359 taken from each section. To detect GFP and YFP, the argon laser $488 \mathrm{~nm}$ was used. For RFP detection, a 360 red diode laser emitting at $561 \mathrm{~nm}$ was used, and blue mCFP was excited using a laser line at $458 \mathrm{~nm}$. 361 Calcein blue staining was detected using the 405-nm laser line. GFP fluorescence was collected between $362 \sim 500-598 \mathrm{~nm}$, airy 1; RFP fluorescence was collected between $\sim 606-654 \mathrm{~nm}$, airy 1; mCFP fluorescence 363 was collected between $\sim 464-500 \mathrm{~nm}$, airy 1 and Calcein blue was collected between $\sim 410-451$. For each 364 image, a corresponding bright field image was captured. The acquired images were processed using 365 Photoshop and ImageJ.

366 To calculate the percentage of Glil+ clones found in both mineralized and non-mineralized 367 fibrocartilage, the border between the zones was identified by Calcein blue signal. Clones that crossed 368 the mineral border were manually counted and their percentage was calculated first for each section, 369 then for each limb and, finally, for each type of enthesis. Data are presented as mean \pm SD.

\section{Acknowledgments}

372 We thank Nitzan Konstantin for expert editorial assistance. We thank Dr. Patrick Tschopp from the 373 Clifford J. Tabin lab, Harvard Medical School, for his assistance in generating Shh KO mice. Special 374 thanks to all members of the Zelzer laboratory for encouragement and advice. 
375 This study was supported by grants from the National Institutes of Health (grant \#R01 AR055580), 376 European Research Council (ERC) (grant \#310098), the Jeanne and Joseph Nissim Foundation for Life 377 Sciences Research, the Y. Leon Benoziyo Institute for Molecular Medicine, Beth Rom-Rymer, the 378 Estate of David Levinson, the Jaffe Bernard and Audrey Foundation, Georges Lustgarten Cancer 379 Research Fund, the David and Fela Shapell Family Center for Genetic Disorders, the David and Fela 380 Shapell Family Foundation INCPM Fund for Preclinical Studies, and the Estate of Bernard Bishin for 381 the WIS-Clalit Program. 


\section{REFERENCES}

Ahn, S. and Joyner, A. L. (2004). Dynamic changes in the response of cells to positive hedgehog signaling during mouse limb patterning. Cell 118, 505-16.

Akiyama, H., Kim, J.-E., Nakashima, K., Balmes, G., Iwai, N., Deng, J. M., Zhang, Z., Martin, J. F., Behringer, R. R., Nakamura, T., et al. (2005). Osteo-chondroprogenitor cells are derived from Sox9 expressing precursors. Proc. Natl. Acad. Sci. U. S. A. 102, 14665-70.

Benjamin, M. and McGonagle, D. (2009). Entheses: tendon and ligament attachment sites. Scand. J. Med. Sci. Sports 19, 520-7.

Benjamin, M., Kumai, T., Milz, S., Boszczyk, B. M., Boszczyk, a a and Ralphs, J. R. (2002). The skeletal attachment of tendons--tendon "entheses". Comp. Biochem. Physiol. A. Mol. Integr. Physiol. 133, 931-45.

Benjamin, M., Toumi, H., Ralphs, J. R., Bydder, G., Best, T. M. and Milz, S. (2006). Where tendons and ligaments meet bone: attachment sites ('entheses') in relation to exercise and/or mechanical load. J. Anat. 208, 471-90.

Blitz, E., Sharir, A., Akiyama, H. and Zelzer, E. (2013). Tendon-bone attachment unit is formed modularly by a distinct pool of Scx- and Sox9-positive progenitors. Development 140, 2680-90.

Breidenbach, A. P., Aschbacher-Smith, L., Lu, Y., Dyment, N. a., Liu, C.-F., Liu, H., Wylie, C., Rao, M., Shearn, J. T., Rowe, D. W., et al. (2015). Ablating Hedgehog Signaling in Tenocytes During Development Impairs Biomechanics and Matrix Organization of the Adult Murine Patellar Tendon Enthesis. J. Orthop. Res. n/a-n/a.

Buckingham, M., Bajard, L., Chang, T., Daubas, P., Hadchouel, J., Meilhac, S., Montarras, D., Rocancourt, D. and Relaix, F. (2003). The formation of skeletal muscle: From somite to limb. $J$. Anat. 202, 59-68. 
Dörfl, J. (1980a). Migration of tendinous insertions. I. Cause and mechanism. J. Anat. 131, 179-95.

Dörfl, J. (1980b). Migration of tendinous insertions. II. Experimental modifications. J. Anat. 131, 22937.

Doschak, M. R. and Zernicke, R. F. (2005). Structure, function and adaptation of bone-tendon and bone-ligament complexes. J. Musculoskelet. Neuronal Interact. 5, 35-40.

Dyment, N. A., Breidenbach, A. P., Schwartz, A. G., Russell, R. P., Aschbacher-Smith, L., Liu, H., Hagiwara, Y., Jiang, R., Thomopoulos, S., Butler, D. L., et al. (2015). Gdf5 progenitors give rise to fibrocartilage cells that mineralize via hedgehog signaling to form the zonal enthesis. Dev. Biol. 405, 1-12.

Galatz, L., Rothermich, S., VanderPloeg, K., Petersen, B., Sandell, L. and Thomopoulos, S. (2007). Development of the supraspinatus tendon-to-bone insertion: localized expression of extracellular matrix and growth factor genes. J. Orthop. Res. 25, 1621-8.

Harfe, B. D., Scherz, P. J., Nissim, S., Tian, H., McMahon, A. P. and Tabin, C. J. (2004). Evidence for an expansion-based temporal Shh gradient in specifying vertebrate digit identities. Cell 118, 517-528.

Kronenberg, H. M. (2003). Developmental regulation of the growth plate. Nature 423, 332-6.

Liu, C.-F., Aschbacher-Smith, L., Barthelery, N. J., Dyment, N., Butler, D. and Wylie, C. (2012). Spatial and Temporal Expression of Molecular Markers and Cell Signals During Normal Development of the Mouse Patellar Tendon. Tissue Eng. Part A 18, 598-608.

Liu, C.-F., Breidenbach, A., Aschbacher-Smith, L., Butler, D. and Wylie, C. (2013). A Role for Hedgehog Signaling in the Differentiation of the Insertion Site of the Patellar Tendon in the Mouse. PLoS One 8, e65411.

Madisen, L., Zwingman, T. A., Sunkin, S. M., Oh, S. W., Zariwala, H. A., Gu, H., Ng, L. L., 
Palmiter, R. D., Hawrylycz, M. J., Jones, A. R., et al. (2010). A robust and high-throughput Cre reporting and characterization system for the whole mouse brain. Nat. Neurosci. 13, 133-140.

Polly, P. D. (2007). Limbs in Mammalian Evolution. Fins into Limbs Evol. Dev. Transform. 245-268.

Razzaque, M. S., Soegiarto, D. W., Chang, D., Long, F. and Lanske, B. (2005). Conditional deletion of Indian hedgehog from collagen type $2 \alpha 1$-expressing cells results in abnormal endochondral bone formation. J. Pathol. 207, 453-461.

Salton, J. A. and Sargis, E. J. (2009). Evolutionary morphology of the tenrecoidea (Mammalia) hindlimb skeleton. J. Morphol. 270, 367-387.

Schwartz, a G., Long, F. and Thomopoulos, S. (2015). Enthesis fibrocartilage cells originate from a population of Hedgehog-responsive cells modulated by the loading environment. Development 142 , 196-206.

Schwartz, A. G., Galatz, L. M. and Thomopoulos, S. (2017). Enthesis regeneration: a role for Gli1+ progenitor cells. Development 144, 1159-1164.

Schweitzer, R., Chyung, J. H., Murtaugh, L. C., Brent, a E., Rosen, V., Olson, E. N., Lassar, a and Tabin, C. J. (2001). Analysis of the tendon cell fate using Scleraxis, a specific marker for tendons and ligaments. Development 128, 3855-66.

Shaw, H. M. and Benjamin, M. (2007). Structure-function relationships of entheses in relation to mechanical load and exercise. Scand. J. Med. Sci. Sports 17, 303-15.

Shwartz, Y. and Zelzer, E. (2014). Nonradioactive in situ hybridization on skeletal tissue sections. Methods Mol. Biol. 1130, 203-215.

Shwartz, Y., Viukov, S., Krief, S. and Zelzer, E. (2016). Joint Development Involves a Continuous Influx of Gdf5-Positive Cells. Cell Rep. 15, 2577-2587.

Snippert, H. J., van der Flier, L. G., Sato, T., van Es, J. H., van den Born, M., Kroon-Veenboer, 
C., Barker, N., Klein, A. M., van Rheenen, J., Simons, B. D., et al. (2010). Intestinal Crypt

Homeostasis Results from Neutral Competition between Symmetrically Dividing Lgr5 Stem Cells.

Cell 143, 134-144.

455

456

Soeda, T., Deng, J. M., de Crombrugghe, B., Behringer, R. R., Nakamura, T. and Akiyama, H. (2010). Sox9-expressing precursors are the cellular origin of the cruciate ligament of the knee joint and the limb tendons. Genesis 48, 635-44.

Stern, T., Aviram, R., Rot, C., Galili, T., Sharir, A., Kalish Achrai, N., Keller, Y., Shahar, R. and Zelzer, E. (2015). Isometric Scaling in Developing Long Bones Is Achieved by an Optimal Epiphyseal Growth Balance. PLOS Biol. 13, e1002212.

Sugimoto, Y., Takimoto, A., Akiyama, H., Kist, R., Scherer, G., Nakamura, T., Hiraki, Y. and Shukunami, C. (2013). Scx+/Sox9+ progenitors contribute to the establishment of the junction between cartilage and tendon/ligament. Development 140, 2280-8.

Vortkamp, A., Lee, K., Lanske, B., Segre, G. V., Kronenberg, H. M. and Tabin, C. J. (1996). Regulation of Rate of Cartilage Differentiation by Indian Hedgehog and PTH-Related Protein. Science (80-. ). 273, 613-622.

Wang, M., Vanhouten, J. N., Nasiri, A. R., Johnson, R. L. and Broadus, A. E. (2013). PTHrP regulates the modeling of cortical bone surfaces at fibrous insertion sites during growth. J. Bone Miner. Res. 28, 598-607. 
Fig.1
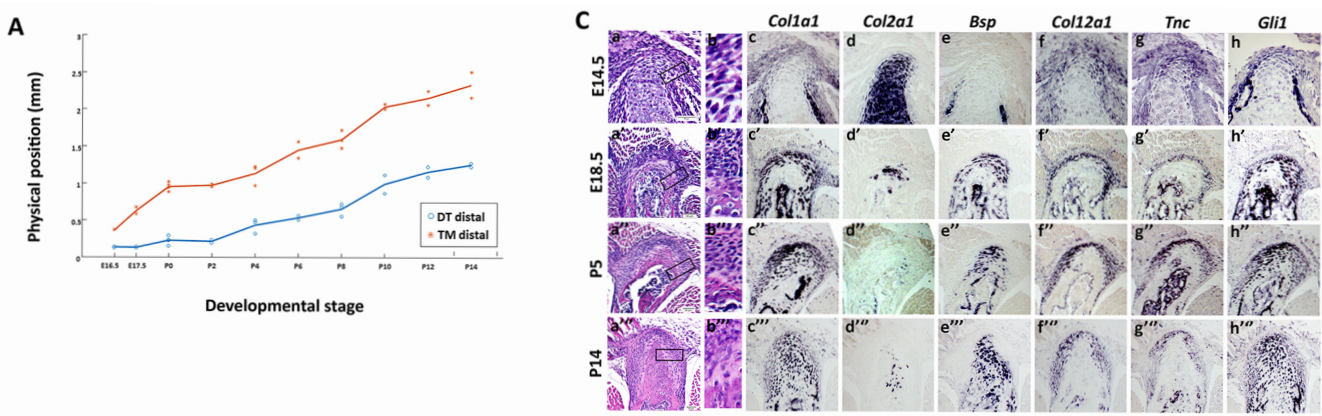

B Deltoid tuberosity

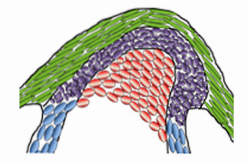

$\Longrightarrow$ Tenocytes

- Perichondrium

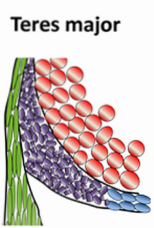

- Attachment

- Chondrocytes 


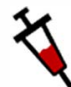

E11.5-E15.5

Fig.2

Gli1-CreER ${ }^{\text {I2 }}$ R26R-td Tomato
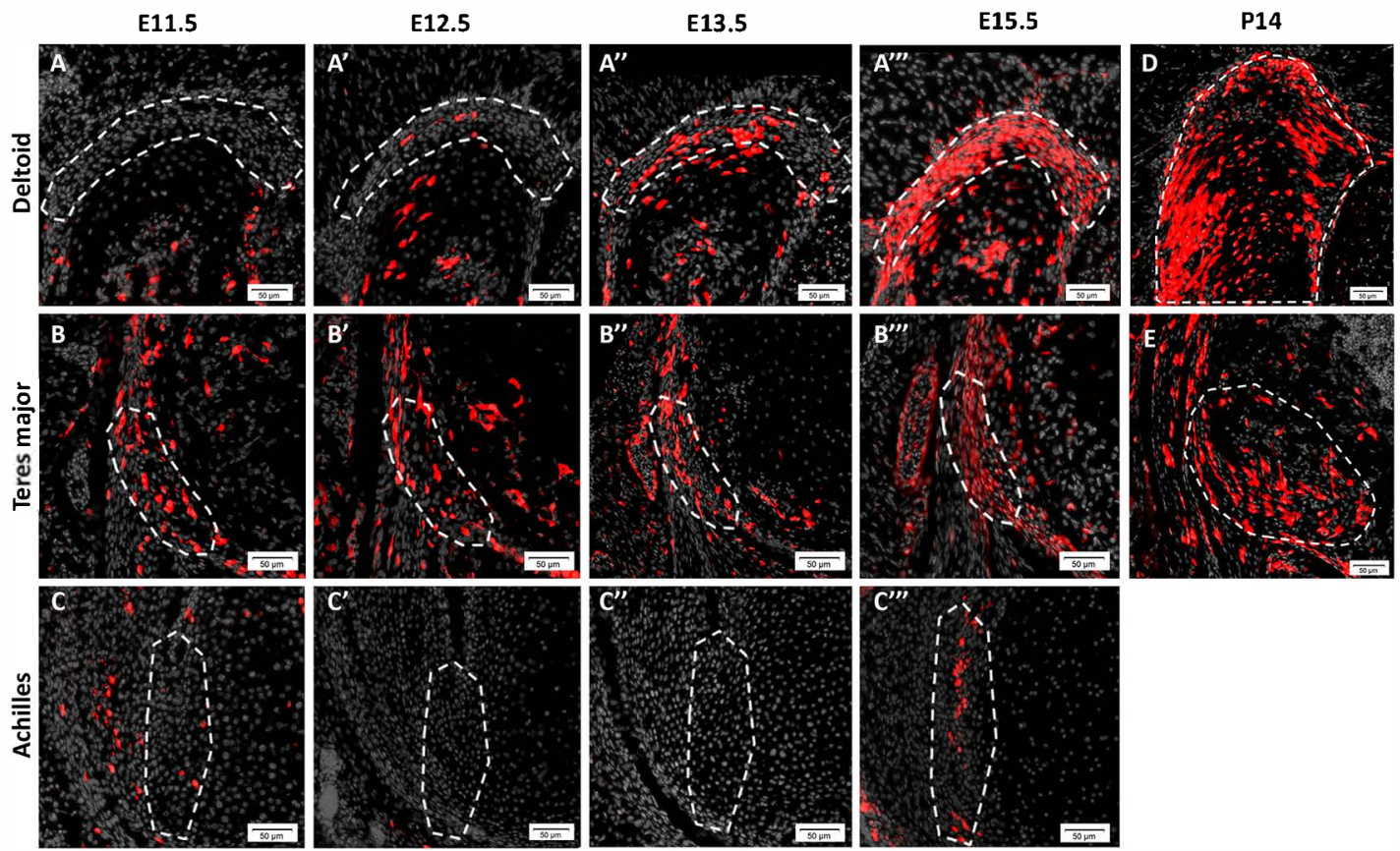
Fig.3

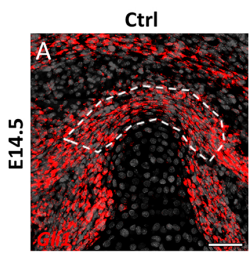

Prx1-Cre-lhh
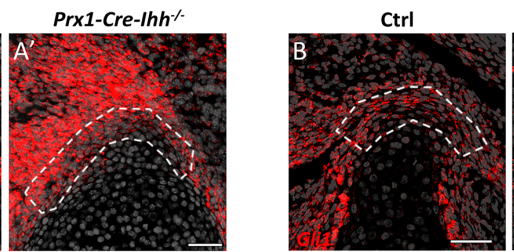

Shh-GFPCre ${ }^{-/}$

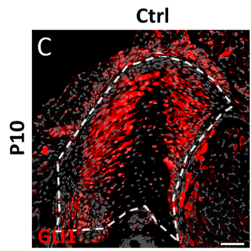

Prx1-Cre-Ihh ${ }^{-/}$

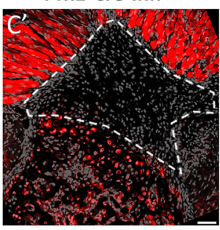

TAM E13.5 $\rightarrow$ E15.5 $\quad$ TAM E13.5 $\rightarrow$ P6
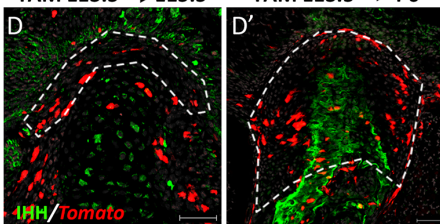
Fig.4
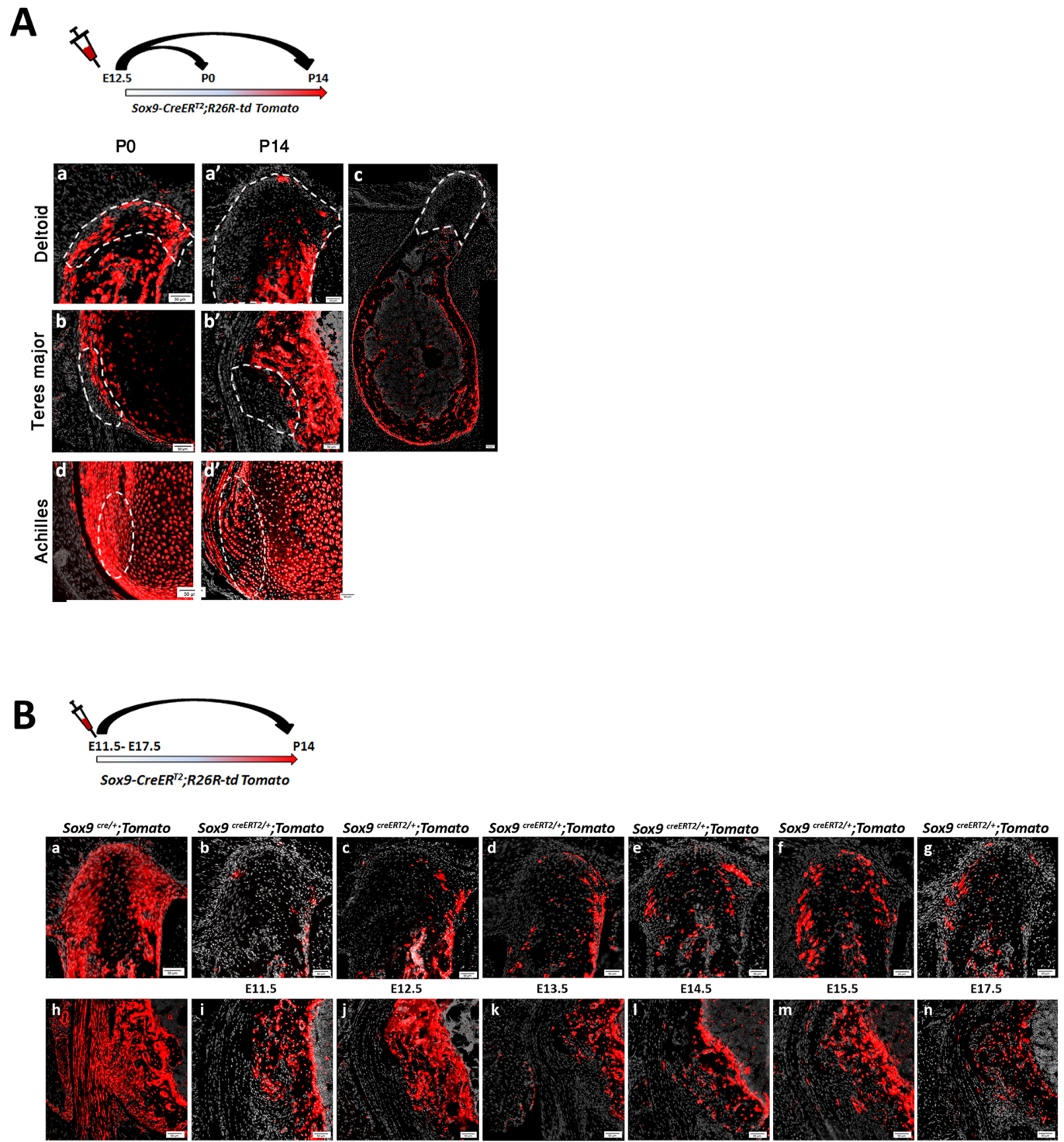
Fig.5
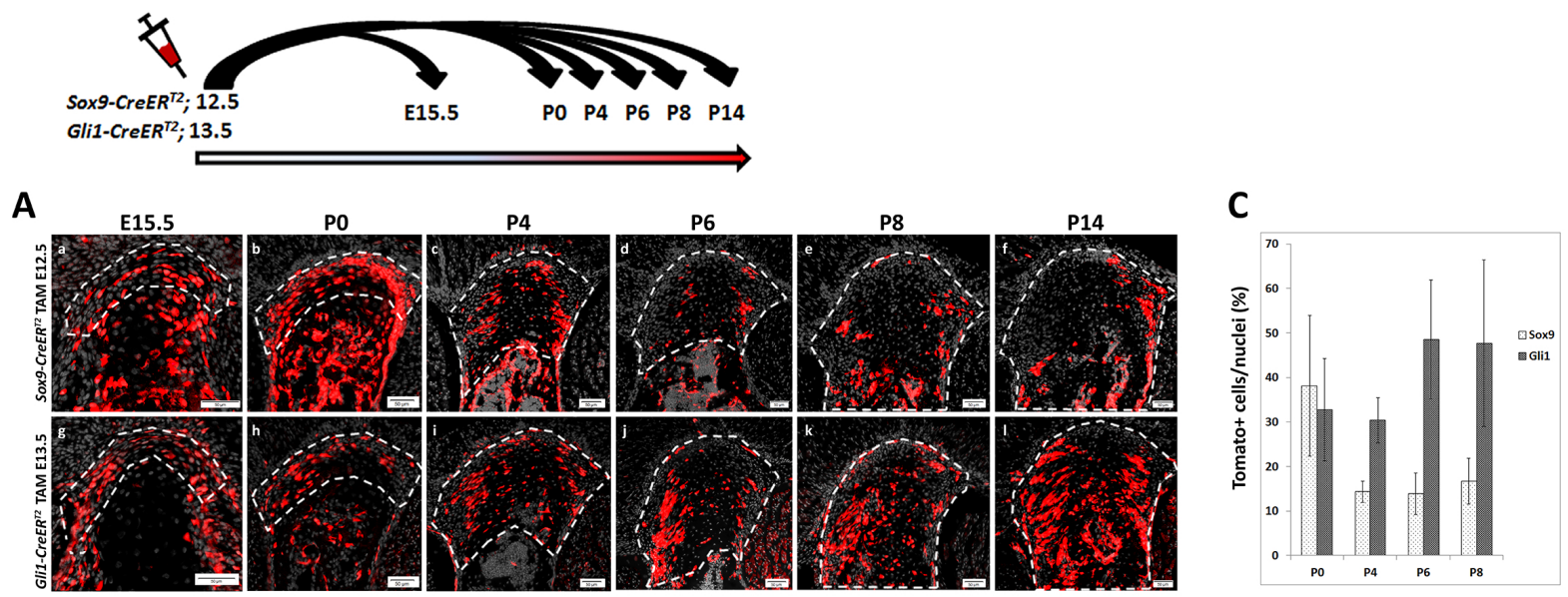

B

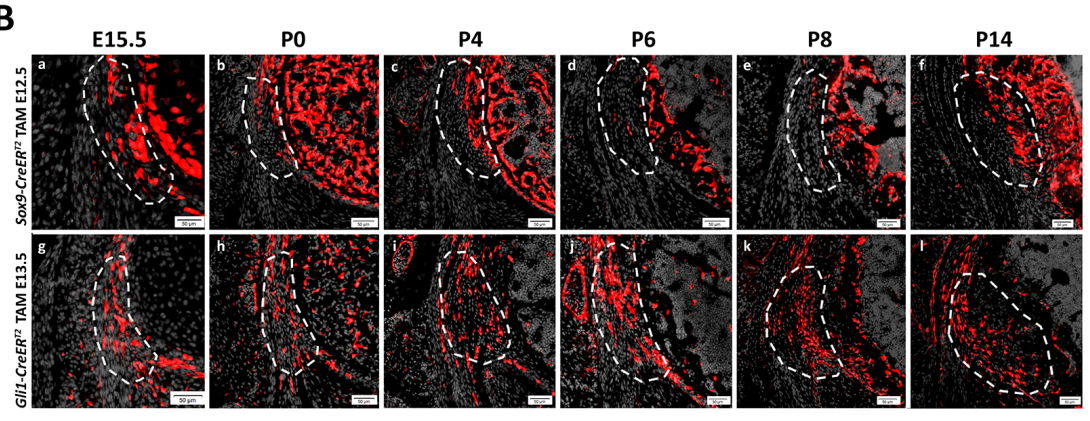

D

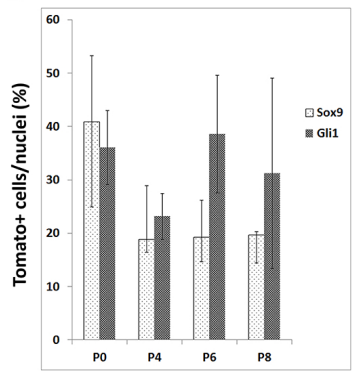




\section{Fig.6}

A

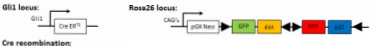

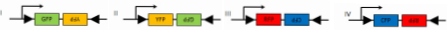

B
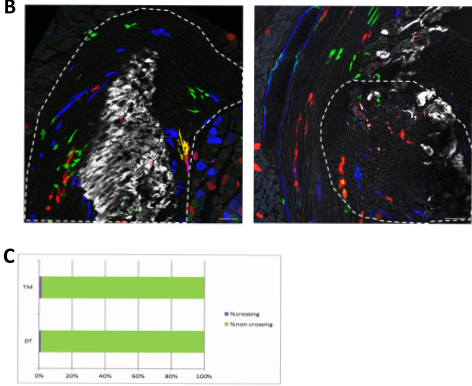


\section{Fig.7}

\section{A. Migratory entheses}

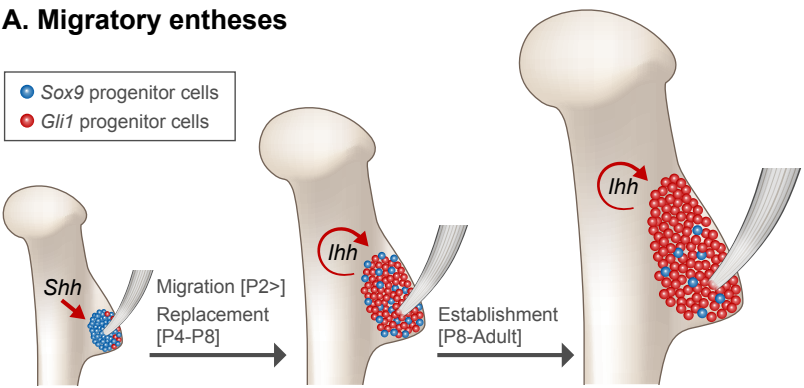

\section{B. Stationary entheses}
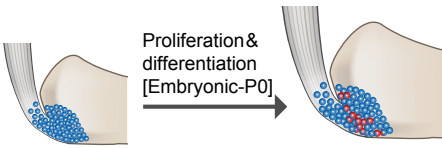

Proliferation \& growth

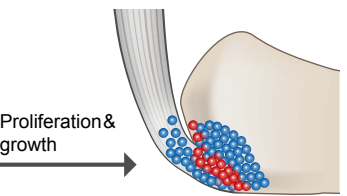




\section{FIGURE LEGENDS}

Figure 1. Some entheses undergo cellular and morphological changes while migrating. (A) Graph showing the physical position of the deltoid tuberosity (DT) and teres major (TM) entheses along the bone throughout development (E16.5-P14), defined as the distance of the element (mm) from a predefined longitudinal origin roughly at the center of the shaft. Positive and negative values correspond to elements that are proximal and distal to the point of origin, respectively. As indicated by the steep slope of the curves, both DT and TM migrate considerably through development. (B) Schematic showing the DT (A) and TM (B) entheses of E14.5-P14 WT mice. [C(a,b) and D(a,b)]: H\&E staining.

h)]: In situ hybridization using digoxigenin-labeled, anti-sense complementary RNA probes for Collal, bars: $50 \mu \mathrm{m}$.

Figure 2. Gli1+ cell lineage contributes to the postnatal enthesis. Pulse-chase cell lineage

experiments using Glil-CreER ${ }^{T 2} ; R 26 R$-tdTomato mice demonstrate the contribution of Glil lineage cells to various entheses. Glil-positive cells were pulsed by a single tamoxifen administration at different time points (E11.5-E15.5) and their descendants were followed to P0 and P14. (A-A',) The DT enthesis was extensively marked after tamoxifen administration at E13.5 and E15.5. (B-B',') tdTomato-positive cells were identified in the TM enthesis regardless of the time of pulsing; however, a stronger signal was seen following tamoxifen administration at E13.5 or later. (C-C',') tdTomatopositive cells were identified in the Achilles tendon enthesis following tamoxifen administration at 
Figure 3. Gli1 expression in migrating entheses is initiated by SHH and maintained by IHH. (A,A') Fluorescent ISH for Glil on sections through the DT of E14.5 control (A) and Prxl-Cre-Ihh $h^{f / f}$ embryos (A') demonstrates that in the absence of IHH signaling, Glil expression in the enthesis is unchanged. (B,B') Fluorescent ISH for Glil on sections through the DT of E14.5 control (B) and Shh ${ }^{\text {Cre/Cre }}$ embryos (B') demonstrates that in the absence of SHH signaling, Glil expression in the enthesis is lost. (C,C') Immunostaining for GLI1 protein in Prxl-Cre-Ihh fff $^{\text {mutant }}\left(\mathrm{C}^{\prime}\right)$ and control (C) DT sections shows that in the absence of IHH, GLI1 protein expression is lost from the DT enthesis. (D,D') Immunostaining for IHH protein on Glil-CreER ${ }^{T 2} ; R 26 R$-tdTomato mice. Tamoxifen was administered at E13.5 and mice were sacrificed at E15.5 (D) or P6 (D'). IHH expression is seen in hypertrophic chondrocytes at E15.5 and in the mineralizing part of the enthesis at P6. Enthesis is demarcated by a dashed line. Scale bars: $50 \mu \mathrm{m}$.

Figure 4. Sox 9 lineage cells of the embryonic enthesis do not contribute to postnatal migrating

entheses. (A) Pulse-chase cell lineage experiment using Sox9-CreER ${ }^{T 2} ; R 26 R$-td Tomato mice demonstrates that Sox9 lineage contributes differently to migrating and stationary entheses. Sox9positive cells were marked at E12.5 by a single tamoxifen administration. At P0, tdTomato-positive cells were identified in migrating DT and TM entheses $(\mathrm{A}(\mathrm{a}, \mathrm{b}))$ as well as in stationary Achilles entheses $(\mathrm{A}(\mathrm{d}))$. At P14, only a few tdTomato-positive cells were identified in migrating entheses $\left(\mathrm{A}\left(\mathrm{a}^{\prime}, \mathrm{b}^{\prime}, \mathrm{c}\right)\right)$, whereas extensive staining was still seen in stationary entheses $\left(A\left(d^{\prime}\right)\right)$. Enthesis is demarcated by a 512 dashed line. Scale bars: $50 \mu \mathrm{m}$. (B) The contribution of Sox9 lineage to the DT and TM entheses was 513 evaluated by crossing Sox9-Cre or Sox9-CreER ${ }^{T 2}$ mice with R26R-tdTomato reporter mice. (Ba,h): The total contribution of the lineage was evaluated by examination of Sox9-Cre;R26R-tdTomato mice at P14. 
516

evaluated by pulse-chase experiments on $\operatorname{Sox} 9-\mathrm{CreER}^{T 2} ; \mathrm{R} 26 \mathrm{R}$-tdTomato mice, in which a single dose of tamoxifen was administered at various stages from E11.5 through E17.5.

Figure 5. Gli1 lineage cells replace Sox9 lineage cells during enthesis maturation. (A,B) Sox9$\operatorname{CreER}^{T 2} ; R 26 R$-tdTomato and Glil-CreER ${ }^{T 2} ; R 26 R$-tdTomato mice were labeled by tamoxifen administration at E12.5 and E13.5, respectively and sacrificed at E15.5-P14. Transverse sections through the DT (A) and TM (B) show an increase in Sox9 lineage cells at E15.5-P0. Between P0 and P14, a continuous decrease in Sox9 lineage cells is seen. Concurrently, Gli1 lineage cell number gradually increased in both DT and TM entheses. By P8, both entheses were populated by Gli1 lineage cells. Enthesis is demarcated by a dashed line. Scale bars: $50 \mu \mathrm{m}$. (C,D) Graphs showing the percentage of Glil-tdTomato and Sox9-tdTomato positive cells in P0, P4, P6, and P8 DT (C) and TM (D) cells.

Figure 6. Cell fate of Gli1+ enthesis progenitors is pre-determined. Pulse-chase cell lineage experiments using Gli1-CreER ${ }^{T 2} ; R 26 R$-Confetti mice, in which Glil+ progenitor cell clones were labeled. Gli1-positive cells were pulsed by a single tamoxifen administration at E13.5 and their descendants were followed to P14. One hour prior to sacrifice, mice were injected with Calcein blue. (A) Schematic illustration of possible Cre recombination outcomes. (B) Multiple clones were identified in the DT enthesis; however, the clones were restricted to either fibrocartilage or mineral fibrocartilage and did not cross the border between the layers. The clones identified in the TM enthesis were restricted to the fibrous part and did not penetrate the bone. (C) Graph showing the percentage of crossing vs. noncrossing clones. Scale bars: $50 \mu \mathrm{m}$.

Figure 7. Migrating entheses develop through template replacement. Schematic illustration of the developmental sequence of migratory and stationary entheses. During embryogenesis, Sox 9 lineage cells form an enthesis template. (A) In migrating entheses, Sox9 lineage cells are replaced by a second population of Glil lineage cells, which eventually form the mature enthesis. Specification of Glil 
539 lineage is regulated by $\mathrm{SHH}$, whereas maintenance of this population is regulated by $\mathrm{IHH}$. (B) In 540 stationary entheses, embryonic Sox9 lineage cells proliferate and differentiate and, eventually, populate 541 the postnatal enthesis structure. During late embryonic development, some Sox9 lineage cells start 542 expressing Gli1.

543 